\title{
SISTEMA HÍBRIDO RENOVÁVEL DE GERAÇÃO DE ENERGIA ELÉTRICA PARA O SEMIÁRIDO
}

\author{
Daniel Albiero, Aline Castro Praciano, Hans Heinrich Vogt, \\ Leonardo de Almeida Monteiro, Erialdo de Oliveira Feitosa \\ Universidade Federal do Ceará (DENA/CCA/UFC) \\ <daniel.albiero@gmail.com>, <alinecastro.praciano@gmail.com $><$ fori@oi.com.br $>$. \\ <aiveca@ufc.br>,<erialdofeitos@gmail.com> \\ DOI: $10.21439 /$ conexoes.v11i1.1067
}

\begin{abstract}
Resumo. O uso de energias renováveis é uma alternativa para a convivência com o semiárido, portanto o manejo de diferentes fontes energéticas pode gerar a sustentabilidade energética dos agricultores desta região. O objetivo deste trabalho foi propor uma Usina Híbrida de Geração de Energia Elétrica através de Fontes Renováveis. Foi utilizada a metodologia de engenharia de projetos para conceituar a Usina Híbrida e através da conceituação foram realizados cálculos preliminares de geração de energia elétrica e custo de investimento. Como resultados foi conceituada e dimensionada a usina híbrida para diversas regiões do semiárido do Estado do Ceará. Concluí-se que este conceito é muito interessante e tecnicamente viável.
\end{abstract}

Palavras-chaves: Energia renovável. Fontes de Energia Sustentabilidade energética.

Abstract. The use of renewable energy is an alternative to living with the semi-arid, so the management of different energy sources can generate energy sustainability of farmers in this region. The aim of this study was to propose a hybrid plant for electricity generation through renewable sources. It was used an engineering methodology to conceptualize the Hybrid Power Plant and through the concept were made preliminary calculations of power generation and investment cost. As a result was conceptualized and sized a hybrid plant to various regions of the state Ceará. According to the results, it was concluded that this concept is very interesting and technically possible.

Keywords: Renewable energy. Source of energy. Energetic sustainability.

\section{INTRODUÇÃO}

Segundo ANDRADE et al. (2006, p. 57) "as regiões semiáridas do globo terrestre se caracterizam pelo déficit hídrico e por um elevado saldo positivo de energia solar. No entanto o nordeste brasileiro tem fatores determinantes específicos tais como solos rasos, alta demanda evaporativa, retirada da cobertura vegetal e organização social. Desta feita torna-se claro que o semiárido brasileiro é uma região muito especial, pois não existe outra no planeta que se aproxime das condições edafoclimáticas, biologicoecológicas e econômico sociais."Para BRASIL (2005) a área classificada como semiárido é de $969.590 \mathrm{~km} 2$, sendo $70 \%$ do Nordeste do Brasil e tem uma população de 53 milhões de habitantes o que corresponde a $34 \%$ da população brasileira
(IBGE - Instituto Brasileiro de Geografia e Estatísticas. 2005).

ANDRADE et al. (2006, p. 138) afirma que "as potencialidades do semiárido devem ser exploradas de forma sustentável e economicamente viável e para isto exigem a compreensão de que a natureza tem que ser respeitada, pois é ela quem deve determinar a forma e a época em que as atividades agrícolas podem ser executadas, portanto é necessário aprender com a diversidade da natureza dessa região, pensando conceitualmente a semiaridez como vantagem e não como desvantagem".

Segundo (ANDRADE; PEREIRA; DANTAS, 2010 , p. 02) "é primordial a definição de um novo modelo de produção agrícola que seja fundamentado nas mudanças tecnológicas de tal forma que a conservação e manejo dos recursos naturais sejam sustentáveis". No 
semiárido não é considerada a capacidade de uso e a aptidão do mesmo (SOUZA; FERNANDES, 2000). "Isto é particularmente grave quando a ação antrópica se dissocia das questões socioeconômicas e ambientais desta região" (ANDRADE; PEREIRA; DANTAS, 2010).

Uma boa alternativa para melhorar a convivência com o semiárido é o uso de energias renováveis (ALBIERO et al., 2014) para a eletrificação rural. A eletrificação rural por intermédio de fontes de energia renováveis na maioria dos casos significa a exigência de sistemas de armazenamento de energia que normalmente utilizam baterias de chumbo-ácido. Isso contribui para a elevação dos custos iniciais de aquisição e instalação. Além dos custos operacionais inevitáveis devido ao ciclo de vida curto das baterias. Essas baterias também se tornaram um perigo adicional ao meio ambiente quando descartadas. Desta forma o custo da eletricidade tornase relativamente elevados e a atração torna-se baixa o que mantem os investidores privados afastados deste tipo de investimento. Uma alternativa que merece ser considerada é o uso da combinação de duas ou mais diferentes fontes de energia configurando os sistemas híbridos de energia (KATTI; KHEDKAR, 2007).

Em função das características climáticas desta região existe uma grande variação nos parâmetros energéticos, principalmente em tempos de seca. Isto significa a necessidade de um manejo energético complementar entre as fontes. Assim vislumbra-se que uma Usina Híbrida é uma boa solução; Principalmente devido a possibilidade de se lançar a energia excedente na rede de distribuição (ANEEL, 2012) o que pode gerar créditos de energia que serão usados em épocas de escassez de recursos. Considerando a filosofia de convivência com o semiárido será utilizada a metodologia da engenharia de projetos que envolve diversas atividades estruturadas para definirem a geração de soluções (BACK, 1983). Este trabalho se atém a concepção de uma Usina Híbrida Renovável.

\section{MATERIAL E MÉTODOS}

A usina híbrida foi conceituada para ser constituída por um sistema de geração de potência eólica, um sistema de geração de potência solar e um sistema de geração de potência química através de biogás. Um novo conceito de turbina eólica para regiões de baixo vento desenvolvido na fase de anteprojeto por Albiero et al. (2014) será a base do sistema em que biodigestores e módulos fotovoltaicos serão integrados.

Para caracterizar de forma adequada no Ceará o conceito da Usina Híbrida proposta, foram escolhidas 8 municípios referentes as 8 macroregiões do Estado. Em cada município será considerada uma propriedade média (20 ha), esta área foi escolhida em função da análise de dados do IBGE - Instituto Brasileiro de Geografia e Estatísticas (2006), que prescreve na região do semiárido esta área para caracterizar uma propriedade média. Os dados dos respectivos municípios escolhidos encontram-se na Tabela 1.

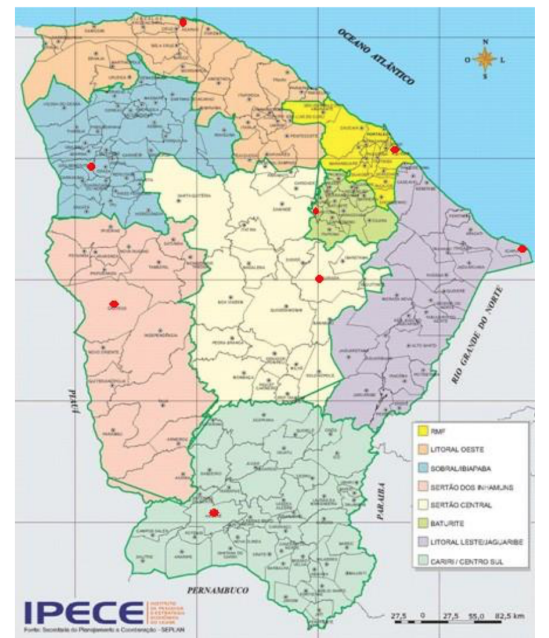

Figura 1: Municípios avaliados e Macro-regiões do Estado do Ceará (IPECE, 2014). Adaptado pelo autor.

Em cada município escolhido foi considerada uma propriedade rural média com 20 ha equipada com rede de energia elétrica monofásica $(220 \mathrm{~V})$ proveniente da distribuidora e as seguintes cargas elétricas:

A potência elétrica gerada pelo sistema eólico proposto e integrante do sistema híbrido é calculada em função das considerações de Albiero et al. (2014) baseia-se nas equações:

$$
\text { Pot }_{\text {eixo }}=P_{m}+P_{\text {eixo }}
$$

Onde: Pot $_{\text {eixo }}$ é ... $C_{p}$ é o coeficiente de potência $(0,31$, ALBIERO et al. 2014); $\rho$ - Densidade do ar $\left(1,23 \mathrm{kgm}^{3}\right) V$ - Velocidade do vento $\left(\mathrm{ms}^{-1}\right) ; R-$ Raio do rotor $(m)$;

$$
P_{m}=P_{g}+P_{d}
$$

Onde: $P_{g}$ é a potência gerada de saída $(W) ; P_{m}$ é a potência Mecânica $(W) ; P_{d}$ é a potência Dissipada (293,4 W), (DAHER, 1997).

Através da distribuição de Weibull é encontrada a frequência em que há vento acima de determinada velocidade de corte (ALVES, 2012). Com este parâmetro é possível determinar quantas horas por dia há vento viável para geração eólica. Considerando a velocidade média anual de vento da região foi feita uma estimativa aproximada da energia elétrica gerada pela turbina 
SISTEMA HÍBRIDO RENOVÁVEL DE GERAÇÃO DE ENERGIA ELÉTRICA PARA O SEMIÁRIDO

Tabela 1 - Dados médios dos Municípios avaliados.

\begin{tabular}{|c|c|c|c|c|c|c|c|c|}
\hline Macro Região & Município & $\begin{array}{l}\text { Veloc. } \\
\text { Média } \\
\text { Vento a } \\
10 \mathrm{~m} \\
(\mathrm{~m} / \mathrm{s})^{*}\end{array}$ & $\begin{array}{c}\text { Estim. } \\
\text { de vento } \\
\text { dia }(24 h) \\
(h)^{* *}\end{array}$ & $\begin{array}{l}\text { Número } \\
\text { médio } \\
\text { Insol. } \\
\text { (h) }\end{array}$ & $\begin{array}{l}\text { Radi. } \\
\text { média } \\
(\mathbf{k W h} / \\
\left.\mathbf{m}^{2}\right)^{*=* *}\end{array}$ & $\begin{array}{c}\text { Cabeças } \\
\text { [Capr] } \\
\text { (Bov.) }\end{array}$ & $\begin{array}{l}\text { Propr. } \\
\text { [Capr] } \\
\text { (Bov.) }\end{array}$ & $\begin{array}{c}\text { Cab/Pr. } \\
\text { [Capr] } \\
\text { (Bov.) }^{+}\end{array}$ \\
\hline Litoral Oeste & Acaraú & 6,3 & 15 & 7 & 5 & $\begin{array}{l}{[372]} \\
(530)\end{array}$ & $\begin{array}{l}\text { [9] } \\
(29)\end{array}$ & $\begin{array}{l}{[41]} \\
(18)\end{array}$ \\
\hline Litoral Leste & Icapuí & 5,9 & 11 & 8 & 6 & $\begin{array}{l}{[216]} \\
(553)\end{array}$ & $\begin{array}{l}{[3]} \\
(41)\end{array}$ & $\begin{array}{l}{[72]} \\
(13)\end{array}$ \\
\hline Ibiapaba & S. Benedito & 5,5 & 18 & 7 & 6 & $\begin{array}{c}{[0]} \\
(393)\end{array}$ & $\begin{array}{c}{[0]} \\
(42)\end{array}$ & $\begin{array}{l}{[0]} \\
(9)\end{array}$ \\
\hline Sertão Central & Quixadá & 4,3 & 9 & 7 & 6 & $\begin{array}{l}{[1044]} \\
(5686)\end{array}$ & $\begin{array}{l}{[69]} \\
(387)\end{array}$ & $\begin{array}{l}{[15]} \\
(14)\end{array}$ \\
\hline Baturité & Aratuba & 5,5 & 17 & 7 & 5 & $\begin{array}{l}{[25]} \\
(305)\end{array}$ & $\begin{array}{c}{[7]} \\
(28)\end{array}$ & $\begin{array}{l}{[3]} \\
(10)\end{array}$ \\
\hline Inhamuns & Crateús & 3,9 & 8 & 7 & 6 & $\begin{array}{c}{[944]} \\
(3592)\end{array}$ & $\begin{array}{l}{[24]} \\
(211)\end{array}$ & $\begin{array}{l}{[39]} \\
(17)\end{array}$ \\
\hline Cariri & Assaré & 2,7 & 10 & 7 & 6 & $\begin{array}{l}{[259]} \\
(2388)\end{array}$ & $\begin{array}{l}{[23]} \\
(152)\end{array}$ & $\begin{array}{l}{[11]} \\
(15)\end{array}$ \\
\hline $\mathrm{ZM}^{1}$ Fortaleza & Aquiraz & 6,3 & 6 & 7 & 5 & $\begin{array}{l}{[52]} \\
(273)\end{array}$ & $\begin{array}{l}{[3]} \\
(15)\end{array}$ & $\begin{array}{l}{[17]} \\
(18)\end{array}$ \\
\hline
\end{tabular}

Tabela 2 - Cargas elétricas das propriedades.

\begin{tabular}{cccc}
\hline Descrição da Carga & $\begin{array}{c}\text { Utilização } \\
\text { diária (h) }\end{array}$ & $\begin{array}{c}\text { Potência } \\
(\mathbf{W})\end{array}$ & $\begin{array}{c}\text { Energia diária } \\
(\mathbf{k W h})\end{array}$ \\
\hline Residência & 12 & 1000 & 12 \\
Sistema Irrigação & 2 & 7360 & 14,7 \\
Máquina Picadora de Forragem & 3 & 1500 & 4,5 \\
Iluminação externa & 6 & 500 & 3 \\
Pequena Oficina Mecânica & 1 & 3000 & 3 \\
Total & - & $\mathbf{7 6 0 0}$ & $\mathbf{3 7 , 2}$ \\
\hline
\end{tabular}

eólica multiplicando-se a velocidade média anual pelo tempo que a turbina opera. Neste trabalho a velocidade de corte será $2,5 \mathrm{~m}$, esta velocidade foi escolhida em função da velocidade média do vento no interior do Estado do Ceará (CEARÁ, 2003). A energia elétrica gerada pelo sistema solar proposto e integrante do sistema híbrido é calculada em função da irradiação solar global do Estado do Ceará e da insolação média no Estado (CEARÁ, 2010). Foi considerada eficiência dos módulos solares de $12 \%$ (PORTAL ENERGIA MAIS 2014). A energia elétrica gerada pelo sistema de biogás proposto e integrante do sistema híbrido é calculado em função dos dados de produção de esterco e de potencial de geração de biogás apresentados por (KUNZ; OLIVEIRA, 2006), que para bovinos de 0,36 m3 biogás/animal/dia. Para caprinos, segundo Quadros et al. (2014) o valor é $0,03 \mathrm{~m} 3 /$ biogás/animal/dia.Um m3 de biogás é equivalente a $5,5 \mathrm{kWh}$ de energia elétrica (QUADROS; VALLADARES; REGIS, 2001). Considerando um gerador utilizando como máquina primária um motor de combustão interna de $5 \mathrm{~kW}$ com efi- ciência média de $35 \%$. O número de cabeças de caprinos/bovinos por propriedade foi estimado em função do número de cabeças e pelo número de propriedades com 20 ha em cada município (IBGE - Instituto Brasileiro de Geografia e Estatísticas, 2006).

\section{RESULTADOS E DISCUSSÃO}

Em função da conceituação foi realizado o desenho conceitual da usina híbrida que é constituída por dois biodigestores tubulares de $6 \mathrm{~m} 3$ (RECOLAST, 2014), 8 placas solares fotovoltaicas de $250 \mathrm{Wp}(\mathrm{BOSCH}, 2014)$ e um sistema de turbinas eólicas com plataforma toroidal de aceleração de rotor "Toroidal Accelerator Rotor Platform" (TARP) com potência total de $1 \mathrm{~kW}$ (ALBIERO et al., 2014), todos estes sistemas são interligados através do acoplamento de 3 inversores de frequência Sanyu SY8000, cada um com potência de saída de 7.5 kW. Os sistemas são apresentados na Figura 2.

A produção de energia elétrica diária da Usina Híbrida em função dos dados da Tabela 1 é apresentada na Tabela 3. 


\begin{tabular}{cccccc}
\hline & \multicolumn{5}{c}{ Tabela3 - Energia produzida diariamente pela Usina Híbrida. } \\
\hline Macro Região & Município & $\begin{array}{c}\text { Energia Eólica } \\
\mathbf{( k W h )}\end{array}$ & $\begin{array}{c}\text { Energia } \\
\text { Solar } \\
\mathbf{( k W h )}\end{array}$ & $\begin{array}{c}\text { Energia Biogás } \\
\mathbf{( k W h )}\end{array}$ & $\begin{array}{c}\text { Total } \\
(\mathbf{k W h})\end{array}$ \\
\hline Litoral Oeste & Acaraú & 16,2 & 7,6 & 42,4 & $\mathbf{6 6 , 2}$ \\
Litoral Leste & Icapuí & 9,2 & 9,1 & 37,6 & $\mathbf{5 5 , 9}$ \\
Ibiapaba & S. Benedito & 11,3 & 9,1 & 17,8 & $\mathbf{3 8 , 2}$ \\
Sertão Central & Quixadá & 1,4 & 9,1 & 30,2 & $\mathbf{4 0 , 7}$ \\
Baturité & Aratuba & 10,7 & 7,6 & 20,3 & $\mathbf{3 8 , 6}$ \\
Inhamuns & Crateús & 0,3 & 9,1 & 40,1 & $\mathbf{4 9 , 5}$ \\
Cariri & Assaré & 0 & 9,1 & 31,5 & $\mathbf{4 0 , 6}$ \\
ZM Fortaleza & Aquiraz & 6,5 & 7,6 & 38,4 & $\mathbf{5 2 , 5}$ \\
\hline
\end{tabular}

Tabela 4 - Valor de investimento para a Usina Híbrida.

\begin{tabular}{cccl}
\hline Sistema & \multicolumn{1}{c}{ Descrição } & $\begin{array}{c}\text { Valor } \\
\text { (RS) }\end{array}$ & Fonte \\
\hline Eólica & 2 Turbinas contrarotativas com TARP & $11.400,00$ & Albiero et al. (2014) \\
Solar & 8 P. Solares Bosch c-Si M60-250 Wp & $9.600,00$ & Mercado Livre (2014) \\
Biogás & 2 Biodigestores tubulares Recolast 6000 & $5.800,00$ & Recolast (2014) \\
Inversores de & 3 Inversores Sanyu SY8000- 7.5 kW & $4.800,00$ & Sanyu (2016) \\
Frequência & Total & $\mathbf{3 1 . 6 0 0 , 0 0}$ & \\
& Ton &
\end{tabular}

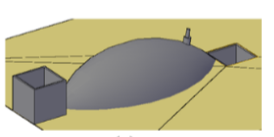

(a)

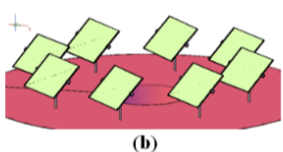

(b)

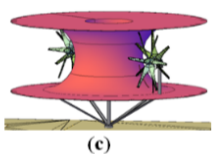

(c)
Figura 2: Partes da Usina Híbrida: (a) Placas Solares; (b) Biodigestores; (c) Turbinas Eólicas

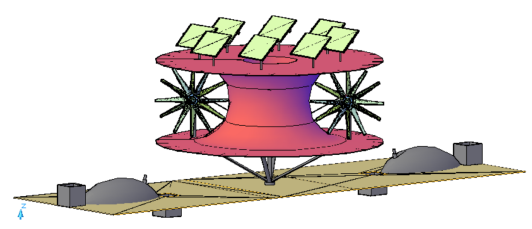

Figura 3: Usina Proposta.

O valor de investimento para se montar a Usina Híbrida é apresentado na Tabela 4. A vida útil considerada é de 15 anos.

Ao analisar-se a Tabela 3 juntamente a Tabela 2, percebe-se que em todos os municípios avaliadas a Usina Híbrida sustenta a demanda. Embora em Assaré, Aratuba e Quixadá, a Usina tenha que operar em carga total. Fato notável é que a Energia de Biogás é a principal fonte de energia em todas as municípios, o que indica a importância da pecuária para a sustentabilidade energética do semiárido. Em Assaré, Quixadá e Crateús a componente Eólica é irrisória o que indica a não necessidade desta fonte para a composição da Usina.

Em termos de manejo operacional das fontes de energia tem-se uma operação complementar entre as fontes eólico/solar e o biogás. Visto que na época da seca, quando a produção animal cai e consequentemente a produção de biogás também, existe forte interação de ventos e insolação que sustentam o sistema.

Em termos de custo de investimento, se for considerado a produção em um mês tem-se que o município com melhor relação custo/benefício é Acaraú com R\$ $15,91 / \mathrm{kWh}$, e a pior é Aratuba com R\$ 27,28/kWh Se o sistema eólico for retirado dos municípios de Assaré, Quixadá e Crateús, o município com melhor relação custo/benefício é Assaré com R\$ 15,43/kWh. Deve-se ressaltar que não foi realizado o estudo de custos variáveis dos sistemas, no entanto não se vislumbra mudança no posicionamento da relação custo/benefício entre os municípios, pois todos os sistemas exigem pouca manutenção. Além dos insumos vento, radiação solar e esterco animal não terem custos.

Outra discussão interessante se refere ao armazenamento da energia excedente que será na rede elétrica da concessionária através do uso dos inversores de frequência para acoplar os sistemas ao sistema da 
SISTEMA HÍBRIDO RENOVÁVEL DE GERAÇÃO DE ENERGIA ELÉTRICA PARA O SEMIÁRIDO

concessionária. No município de Acaraú, uma propriedade média que tenha a Usina terá uma sobra de 29 kWh/dia de energia. Atualmente é possível lançar esta energia excedente na rede de distribuição da concessionária o que gera créditos para serem usados mais tarde (ANEEL, 2012). Este fato possibilita que a propriedade evite ter um sistema de armazenamento de energia próprio através de baterias, solução onerosas e prejudicial ao ambiente. Desta forma esta energia excedente em vez de ser armazenada na propriedade é armazenada na forma de água em hidrelétricas ou petróleo nas termoelétricas, pois esta usinas convencionais economizarão estes insumos em sua operação.

\section{CONCLUSÕES}

A Usina Híbrida atendeu a demanda de energia de uma propriedade média de 20 e gerou uma representativa energia excedente em 4 dos 8 municípios estudados.

O manejo operacional da Usina é otimizado em função das especificidades climáticas do semiárido, principalmente em relação a complementaridade entre fontes energéticas renováveis nos períodos de seca.

O custo de investimento é relativamente baixo e o valor do investimento do $\mathrm{kWh}$ considerando um mês de operação é muito interessante. A possibilidade de lançar o excedente de energia na rede torna muito viável este conceito de Usina Híbrida de Fontes Renováveis.

\section{REFERÊNCIAS}

ALBIERO, D.; DAHER, S.; MONTEIRO, L. de A.; CANAFÍSTULA, F. J. Turbina eolica para agricultura familiar do semiarido com inovac? es tecnologicas para baixas velocidades de vento. Revista Ciência Agronômica, Universidade Federal do Ceará, Centro de Ciências Agrárias, v. 45, n. 1, p. 186, 2014.

ALVES, J. J. A. Regionalização do potencial eólico no estado do ceará (potential wind regionalization in the ceará state). Revista Brasileira de Geografia Física, v. 5, n. 2, p. 332-345, 2012.

ANDRADE, A. d.; SOUZA, E. d.; SILVA, D. d.; SILVA, I.; LIMA, J. R. S. Produção animal no bioma caatinga: paradigmas dos' pulsos-reservas'. Revista Brasileira de Zootecnia, v. 35, n. ssupl. especial, 2006.

ANDRADE, E. M. d.; PEREIRA, O. J.; DANTAS, F. Semiárido e o manejo dos recursos naturais: Uma proposta de uso adequado do capital natural. Fortaleza: UFC, 2010.
ANEEL, A. N. D. E. E. Resolução Normativa

REN-482. Brasília: , 2012. Disponível em: <http: //http://www.aneel.gov.br/cedoc/ren2012482.pdf>

BACK, N. Metodologia de projeto de produtos industriais. : Guanabara Dois, 1983.

BOSCH. Bosch Solar Energy. 2014. Disponível em: $<w w w . b o s c h-s o l a r e n e r g y . c o m>$

BRASIL, M. d. I. e. d. M. A. Nova delimitação do semi-árido Brasileiro. Brasília: , 2005. Disponível em: <www.mi.gov.br >

CEARÁ, G. d. E. Atlas do Potencial Eólico do Ceará. Fortaleza: , 2003. Disponível em: <http://www25.ceara.gov.br>

Relatório de Energia Solar. Fortaleza: , 2010. Disponível em: <http://www25.ceara.gov.br>

DAHER, S. Um sistema baseado em gerador de indução trifásico para aproveitamento da energia eólica. Universidade Federal do Ceará, 1997.

IBGE - Instituto Brasileiro de Geografia e Estatísticas. Censo. Brasília: , 2005. Disponível em: $<w w w . i b g e . g o v . b r>$

. Censo Agropecuário. Brasília: , 2006.

Disponível em: <www.ibge.gov.br>

IPECE. Caracterização Territorial. 2014. Disponível em: <http://www2.ipece.ce.gov.br/atlas/>

KATTI, P. K.; KHEDKAR, M. K. Alternative energy facilities based on site matching and generation unit sizing for remote area power supply. Renewable Energy, Elsevier, v. 32, n. 8, p. 1346-1362, 2007.

KUNZ, A.; OLIVEIRA, P. A. V. d. Aproveitamento de dejetos de animais para geração de biogás. Revista de Política Agrícola, v. 15, n. 3, p. 28-35, 2006.

PORTAL ENERGIA MAIS. Eficiência de Células Solares. 2014. Disponível em: <http://www. portal-energia.com/principais-tipos-de-celulas-। fotovoltaicas-constituintes-de-paineis-solares/>.

QUADROS, D. G. de; VALLADARES, R.; REGIS, U. Aproveitamento dos dejetos de caprinos e ovinos na geração de energia renovável e preservação do meio ambiente. Núcleo de Estudos e Pesquisas em Produção Animal, Universidade do Estado da Bahia - Salvador, 2001.

RECOLAST. Produtos: Biodigestores. 2014. Disponível em: <http://www.recolast.com.br/> 
SANYU. Produtos: Inversores de Frequência. 2014.

Disponível em: <http://www.sanyuacdrive.com/>

SOUZA, E. d.; FERNANDES, M. R. Sub-bacias

hidrográficas: unidades básicas para o planejamento

e a gestão sustentáveis das atividades rurais. Informe

Agropecuário, Belo Horizonte, v. 21, n. 207, p. 15-20,

2000. 